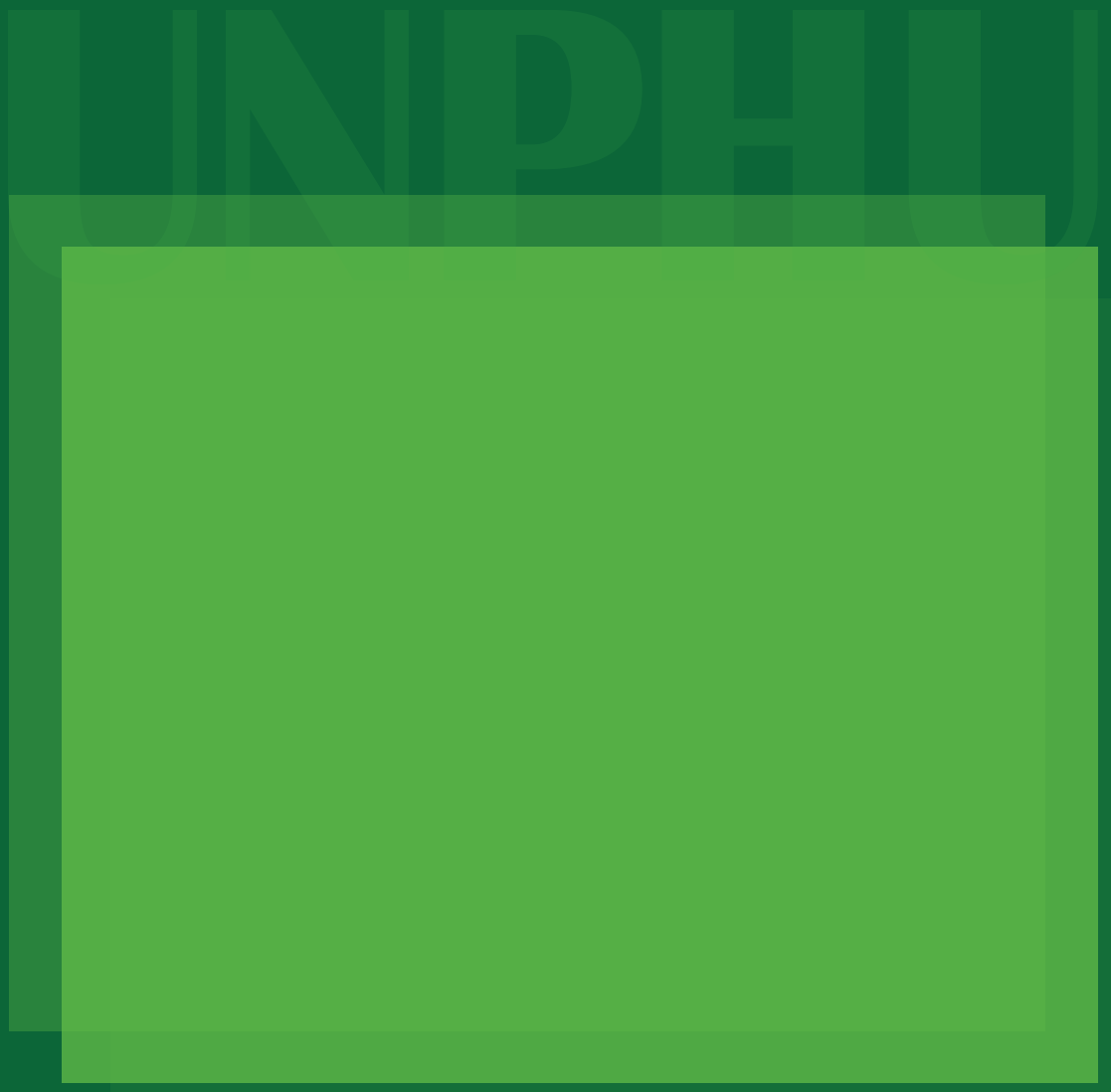




\title{
Responsabilidad Ambiental Universitaria: Una experiencia para compartir Universidad Nacional Pedro Henríquez Ureña
}

\author{
Dolly Martínez \\ Directora Ambiental, docente y miembro de la Unidad de \\ Calidad Académica de la Escuela de Ingeniería Civil de la UNPHU. \\ dmartinez@unphu.edu.do
}

Fecha de recepción: 27 de diciembre de 2017

Fecha de aceptación: 13 de abril de 2018.

Favor de citar este artículo de la siguiente forma:

Martínez D. (2018) Responsabilidad Ambiental Universitaria: Una experiencia para compartir.

Revista AULA. Vol. 62. Número 2, enero-junio 2018. Santo Domingo: Amigo del Hogar.

\section{RESUMEN}

La Responsabilidad Ambiental Universitaria, concepto en proceso de construcción a nivel mundial, es el centro de interés de este artículo en el que se exploran los alcances de la misma, beneficios no considerados usualmente y fortalezas a destacar. Incluye además la experiencia desde la UNPHU, primera universidad privada de Santo Domingo, en República Dominicana, verificándose beneficios como el fortalecimiento de la imagen institucional, las bondades de un voluntariado estudiantil robusto y la alta valoración que los voluntarios de su programa ambiental tienen que este ejercicio aporta a su formación profesional. Al final se comparten algunas reflexiones que pueden servir para realizar investigaciones más profundas en áreas específicas del tema.

Palabras clave: educación, medioambiente, responsabilidad ambiental universitaria, responsabilidad social, universidad, UNPHU.

\begin{abstract}
The University Environmental Responsibility, a concept in the process of construction worldwide, is the center of interest of this article in which its scope is explored, having in mind benefits not usually considered and strengths to be highlighted. It also includes the experience from UNPHU, the first private university in Santo Domingo, Dominican Republic, verifying advantages such as the strengthening of the institutional image, the benefits of a robust student volunteering and the high assessment that the volunteers of its environmental program have on how this exercise contributes to their professional training. In the end, some reflections are shared that can be used to carry out more indepth investigations in specific areas of the topic.
\end{abstract}

Keywords: education, environment, social responsibility, university environmental responsibilities, university, UNPHU. 


\section{Responsabilidad Ambiental Universitaria: Una experiencia para compartir Universidad Nacional Pedro Henríquez Ureña}

\section{Introducción}

La Responsabilidad Ambiental Universitaria (RAU), deviene del concepto de Responsabilidad Social aplicado a las universidades y enfocado en el aspecto ambiental, tocando cada uno de los ejes de la universidad. Pudiese considerarse que la RAU es el mismo tipo de responsabilidad social que la que ejercen el resto de las empresas, pero este planteamiento obvia la naturaleza misma de las universidades, sus objetivos y la manera en que se manifiesta, así como los indicadores en que puede medirse, como podrá apreciarse en el desarrollo del presente artículo. Así mismo, cabe indagar si realmente es beneficiosa y compatible con la razón de ser de la universidad y de manera puntual, verificar cómo se desarrolla y los posibles beneficios que ha reportado su práctica en la Universidad Nacional Pedro Henríquez Ureña (UNPHU), de República Dominicana.

\section{Analizando diferencias y enfoques}

Para distinguir la responsabilidad social que realiza una empresa de la que atañe a una universidad, es necesario comparar definiciones breves de cada una.

Según Prezioza (2005), quien hace un exhaustivo análisis de la definición de la RSE en diferentes aspectos, concluye explicando el concepto de la siguiente forma:

Es el carácter ético de la actuación de la empresa vista desde su relación con la sociedad, anclada en una cierta autoría organizacional análoga a la autoría personal y en su desempeño racional y diligente, respetuoso y proactivo en el desarrollo humano en sociedad. (p. 55).
La RSU es una política de mejora continua de la universidad, hacia el cumplimiento efectivo de su misión social mediante cuatro procesos:

- Gestión ética y ambiental de la institución,

- Formación de ciudadanos responsables y solidarios,

- Producción y difusión de conocimientos socialmente pertinentes,

- Participación social en promoción de un

Desarrollo más humano y sostenible.

(Vallaerys, 2008, p. 2009).

Es el desarrollo de su gestión ambiental donde se manifiesta la responsabilidad social en la universidad de una forma particular al propiciar un ejercicio distinto del que atañe a las empresas en general. No la ejerce para buscar un beneficio económico o mejorar su imagen con los steakholders, lo hace para el bien de la comunidad y el medioambiente. Esta responsabilidad ambiental, según señalan Senior, A., y Narváez, M., y Fernández, G., y Revilla, J. (2007, p. 4), implica el que se reduzcan los daños y se amplíen los beneficios al medioambiente y resolver problemas ambientales de la comunidad. Las Instituciones de Educación Superior (IES) abarcan tres aspectos fundamentales, como son el formativo, el de investigación y desarrollo, así como el de vinculación y trabajan en dos vías, tanto hacia la comunidad educativa en sí misma como hacia la exterior, reflejada como sociedad en general y la comunidad que rodea a la institución. Así mismo se manifiesta en cinco ejes fundamentales, como son las asignaturas ambientales, los trabajos de investigación, las ofertas de 
postgrado, trabajos de grado y la gestión misma de la institución, (Eschenhagen, 2011).

La práctica de esta RAU, para ser real y congruente, debe resultar de escuchar las tendencias, percatarse de la forma de pensar de la comunidad, de los consumidores, para poder acertar con los objetivos trazados. Aspectos como el de la energía o el voluntariado, adquieren dimensiones más amplias que en las instituciones tradicionales, ya que, en el caso de la energía, no se trata de un ahorro económico, sino más bien de un ejercicio congruente con la filosofía y objetivos institucionales, o lo que llamamos, enseñar con el ejemplo. Así mismo, el aspecto de voluntariado, incluye metas más allá de la sola satisfacción personal del voluntario. Implica el desarrollo integral del estudiante al involucrar competencias y habilidades cuyo desarrollo revela un egresado con mayores capacidades para dar soluciones a las problemáticas de la sociedad; de desarrollarse plenamente como ser humano; de tener mayor probabilidad de éxito al tener una vida más equilibrada.

Al desarrollar las universidades una labor de responsabilidad ambiental que sea parte de su misión institucional y que se manifieste en un programa que refleje sus ejes estratégicos, inciden no sólo en la reducción o eliminación del impacto de las actividades cotidianas sobre el medioambiente, sino también en una mayor sensibilidad en los temas ambientales. Así mismo, en el aspecto moral y cívico de la persona, y en un desempeño más efectivo en las labores gestión de la institución, lo que se traduce en una mejoría del resultado económico. Además, provee conocimientos que permiten aumentar las capacidades de la población para dar respuesta y soluciones a las situaciones que les afectan negativamente, permitiendo con esto el empoderamiento de las comunidades.

Pudiese pensarse que la RAU es una especie de juego altruista en el que sólo pocas univer- sidades pudiesen participar, pero la economía global ha cambiado paradigmas. Como indica Marginson (2004) citado por Takayanagui, A. D. y de la Fuente, J. R. (2015), el poseer conocimiento e información, son algunos de los aspectos que aumentan la productividad en el área económica. Siendo uno de los objetivos fundamentales de las Instituciones de Educación Superior (IES) el ser formadoras del conocimiento, son estos ejes sobre los que gira la producción y divulgación de este conocimiento, convirtiéndose en referentes a la hora de trazar nuevas líneas económicas y de producción, modelos de conducta a seguir, innovación, áreas de saberes a desarrollar y proporcionar buenas acciones a replicar.

Así mismo, De Sousa (2007) señala que "Desde el inicio de la década de 1990, los analistas financieros han llamado la atención hacia el potencial que tiene la educación para transformarse en uno de los más vibrantes mercados del siglo XXI.", (p.34, 35), lo que se manifiesta hoy día y sin embargo esto no las distrae de su naturaleza esencial. Al verlas como empresas capitalizadoras del conocimiento y generadoras de ingresos en función de este conocimiento no se puede olvidar su vocación.

El Informe de Seguimiento de la Educación en el Mundo 2016, de la Organización de las Naciones Unidas para la Educación, la Ciencia y la Cultura (UNESCO), señala que "La nueva Agenda 2030 para el Desarrollo Sostenible asocia en un solo marco los objetivos de desarrollo mundial y los objetivos medioambientales", (2016, p.5). Esto destaca la importancia que debe tener el aspecto medioambiental en la agenda país y el currículo. Y dado que los efectos del cambio climático se revelan cada vez con mayor frecuencia y fuerza a nivel mundial, así como la gran repercusión que en la economía de los países provocan los desastres naturales, la forma en que las IES manejen el tema ambiental dentro y fuera del campus 
universitario, justifica el papel preponderante que debe tomar este tema en el desempeño de sus actividades. Por esto, la RAU no solamente aporta soluciones dentro de la propia gestión institucional, (manejo de residuos, emisiones de gases, etc.), sino que es parte de su deber ofrecer soluciones a las situaciones que entren en conflicto con el adecuado manejo ambiental de las acciones humanas, innovar y crear conocimiento nuevo que permita un desarrollo económico sostenible.

En la actualidad se están generando propuestas nuevas, como por ejemplo la que plantea la Economía Circular, que están en consonancia con lo que indica la Agenda 2030 para el Desarrollo Sostenible, pues es más que evidente que no es posible continuar generando riqueza con el actual modelo productivo. Se hace urgente, estudiar y plantear nuevos paradigmas económicos para reorientar la productividad de la sociedad.

\section{Beneficios de la RAU}

Entre los múltiples beneficios que aporta a las universidades el ejercicio de su responsabilidad ambiental, uno que pocas veces se menciona es el que se crea como parte de los activos intangibles. El fortalecimiento institucional es uno de ellos y no debe obviarse alegando que el beneficio económico no es de los objetivos principales de las IES, ya que efectivamente, la hace más sostenible y le permite mejorar su alcance. Fernández García (2013) indica que la sostenibilidad tiene una triple dimensión: económica, social y medioambiental, enfatizando la incongruencia entre una empresa sostenible y que a la vez, afecte negativamente el medioambiente. Por otro lado, García-Ayuso y Larrinaga (2004), señalan las ventajas de los activos intangibles y enfatizan en que los medioambientales, dada su naturaleza, son los más difíciles de integrar, ignorándose su in- clusión al momento de declarar o reportar este tipo de activos. Así mismo, al incluir la imagen de la empresa como un valor intangible, que se ve fortalecida por su desempeño en diferentes ámbitos como el ambiental, provoca beneficios como por ejemplo el de mayor lealtad de los clientes. Esto se ve corroborado por los resultados de los estudios realizados por Cone Communications a nivel mundial, en el 2013, en alianza con ECHO y junto a Equibity en 2015, en cuanto a que el $88 \%$ de los consumidores o clientes son más leales a las empresas que apoyan problemas sociales y medioambientales, el 90\% tiene mayor probabilidad de confiar en ellas y el $93 \%$ tiene una imagen más positiva. En el estudio del año 2009, en alianza con Equibity y aplicado a 9,709 personas en 9 de los países más grandes del mundo, muestran la gran importancia que le dan los encuestados al involucramiento de las empresas en el aspecto medioambiental; que les interesa saber más sobre este desempeño por las redes sociales y que su presencia, a la hora de elegir, se orienta hacia las empresas que tienen una mayor responsabilidad social. Por supuesto que sería importante contextualizar estos resultados en la situación específica de las universidades de nuestro país y región, a través de estudios de investigación, aunque estos interesantes datos proporcionan una tendencia mundial que es difícil de obviar.

Lo cierto es que un correcto desempeño en la RAU se traduce en un valor agregado a ser considerado por la institución, a la vez que crea experiencias únicas en el estudiantado, entre otros beneficios que producen una propuesta educativa diferenciadora y que es apreciada por los bachilleres que buscan y sopesan opciones entre las universidades para realizar su carrera universitaria. Esta ventaja competitiva se manifiesta con más fuerza en la medida en la que las IES sean más transparentes con las informaciones que ofrecen al público, lo cual es necesario y casi imprescindible, sobre todo al tratar con instituciones cuyos valores éticos deben mani- 
festarse con los hechos y ser percibidos fácilmente a través de sus medios de comunicación, no solo por medio de esporádicos reportajes en estos, sino por la evidencia de las actividades y acciones realizadas a lo largo del año así como la publicación de sus informes anuales. El joven actual percibe fácilmente la diferencia de un "producto" creado para reflejar una realidad que no existe, y una oferta de vida congruente con sus valores.

Otro de los beneficios para la IES es que posea una imagen fortalecida por su desempeño exitoso en cuanto a su responsabilidad ambiental, estrechando vínculos no solamente con sus estudiantes o los futuros a ingresar, también con el Estado y otras empresas, nacionales e internacionales, además de obtener mejor resultado con las acreditadoras. Es cada vez más valorado este aspecto, por empresas que buscan aliarse o invertir en IES con una adecuada RAU. Y este camino de crear alianzas, parte del ODS número 13, es otro factor de éxito que incide en la sostenibilidad de la universidad.

\section{Una experiencia a compartir}

La UNESCO a través del Instituto Internacional para la Educación Superior en América Latina y el Caribe IESALC, indica que la RAU "comienza en su gestión ambiental interna". Desde la experiencia de la UNPHU, su responsabilidad ambiental se ha ido desarrollando con los años en aspectos diversos de la vida universitaria. Tuvo sus inicios hace diez años, cuando aún no se tenía un concepto amplio y definido de RAU en el país y apenas se hablaba de la conciencia "verde" o medioambientalista. Con el tiempo, ha ido infiltrándose en las diferentes áreas de la academia.

En el aspecto energético, fundamental para el desarrollo de las actividades, se ha optado por la energía solar dentro de las renovables, iniciando la primera etapa en el campus de Santo Domingo con la instalación de 1,963 paneles solares que impiden la emisión de al menos 2,800 kilogramos diarios de $\mathrm{CO} 2$ a la atmósfera. Esta decisión es sumamente importante ya que es congruente con el Objetivo de Desarrollo Sostenible número siete y la Estrategia Nacional de Desarrollo (END 2030) del país.

Según indica el secretario general de la ONU, en el informe presentado a mediados de este año: "La proporción de energía renovable respecto al consumo final de energía aumentó ligeramente de 2012 a 2014, del 17,9\% al 18,3\%. La mayor parte de ese aumento correspondió a electricidad renovable procedente de energía hidroeléctrica, solar y eólica. ', (2017, p. 11). Esto muestra una tendencia de crecimiento que se manifiesta y permanece a nivel mundial y en nuestro país, de manera específica en la energía solar. Por otra parte, el informe del Worldwatch Institute, (2015), pone de manifiesto el enorme potencial que posee este tipo de energía renovable para República Dominicana, haciendo una afirmación que pudiese parecer un sueño al señalar que la demanda de energía eléctrica del país puede ser satisfecha con energías renovables y "unos 86 kilómetros cuadrados de paneles solares fotovoltaicos podrían satisfacer la producción de energía total del país en 2010", (p. 57). A pesar de todo, el incremento en la cantidad de los proyectos implementados por el gobierno así como de instituciones públicas y privadas que se acogen a este tipo de energía, en los últimos años, es esperanzador.

Por otra parte, se trabaja con combustible diesel de bajo contenido de azufre, reduciendo en alrededor de un 30\% las emisiones de materia particulada, para plantas eléctricas y vehículos institucionales, proyectando para el año 2018, que la reducción sea de alrededor de un $50 \%$. Este aspecto es muy apreciado, ya que beneficia la calidad del aire del campus, independientemente de la gran vegetación que 
posee y de los muros "verdes" de bambú, que atraviesan longitudinalmente el campus y se preservan por ser grandes captadores de $\mathrm{CO} 2$. Así mismo la eliminación gradual del uso de mercurio en las prácticas docentes, entre otras acciones que se realizan para apoyar los objetivos y acciones del país para dar cumplimiento a acuerdos internacionales.

Existe también un programa para reutilizar los equipos tecnológicos que ya no tengan utilidad en alguna de las áreas administrativas, haciéndolos llegar a otros usuarios de la institución, lo que prolongará el tiempo de uso del equipo, evitando descartarlo.

En la gestión de residuos, se ha iniciado un plan de separación desde la fuente, con la inclusión de contenedores diferenciados para separar en una primera etapa algunos tipos de desechos como botellas plásticas y latas, papel y cartón, de otros residuos, aunque algunos materiales como los metales y el vidrio tienen otro sistema de acopio. Cabe resaltar la labor que se realiza desde la unidad de separación de residuos del tipo biológico peligroso infeccioso, basado en el código de la Lista Europea de Residuos (LER), en las escuelas de Odontología y Veterinaria, labor que se ampliará próximamente a otras escuelas. La disposición final de estos residuos es realizada mediante una empresa especializada en esta labor y autorizada por el Ministerio de Medioambiente y Recursos Naturales. Una ventaja adicional es que permite a los estudiantes vivenciar los beneficios que esta buena práctica ofrece.

El aspecto de voluntariado medioambiental es una de las fortalezas de la RAU dentro de la UNPHU y dentro de la estructura organizacional, se encuentra el Programa 'Pienso en Verde" que pertenece a la Dirección Medioambiental, teniendo como una de sus áreas de acción la del voluntariado estudiantil medioambiental. El voluntariado es definido por la Ley 61-13 “como el mecanismo de parti- cipación mediante el cual las personas motivadas por un espíritu altruista, se articulan a fin de poner en acción los valores de cooperación y solidaridad en beneficio de la comunidad". El programa de Voluntario de las Naciones Unidas (2011), señala como los principios básicos que lo rigen "un deseo de contribuir al bien común, libremente y con espíritu de solidaridad, sin esperar a cambio ninguna recompensa material.". Indican también que otros valores característicos del voluntariado son el bienestar social e individual, así como la inclusión y el auto gestionarse, suscitando una satisfacción generada por la mejoría que se ofrece a la vida de lo que se apoya.

Los autores Vecina, Chacón y Sueiro, (2009), en el estudio realizado a 673 voluntarios, concluyen que .... la satisfacción de los voluntarios de la muestra incluye tres dimensiones relacionadas con la forma de gestionar de la organización, con las tareas realizadas y con satisfacción de las motivaciones". (p. 117). Esta satisfacción es fundamental a la hora de lograr el involucramiento y la permanencia de los voluntarios. Haciendo referencia a Penner (2002), señalan que la manera en que se relaciona la institución con el voluntario influye en su grado de integración, resaltando la importancia de la satisfacción organizacional sobre todo al implicar reconocimiento por parte de la misma, corroborado esto por el VNU (2011). De igual manera, al estar la satisfacción de las tareas relacionada con su naturaleza misma, el estudiante que se involucra en el voluntariado mediante actividades y labores que contribuyen al medioambiente, percibe de manera directa el aporte positivo que su labor ejerce. Chacón y Dávila (2001) en un estudio aplicado a 323 voluntarios para determinar la diferencia entre el perfil motivacional de los socio asistenciales y medioambientales, indican que estos últimos se encuentran más motivados, toda vez que desean manifestar con mayor intensidad sus valores personales relacionados con su inclinación al medioambiente. Siendo la formación integral 
y la sensibilidad medioambiental, de los objetivos a lograr, es comprensible que el crear los espacios para el desarrollo de estos sea prácticamente una obligación. Dentro de las labores que se realizan en la UNPHU en ese sentido, está lo relacionado con las jornadas de siembra, de limpieza de playas, actividades de ayuda comunitaria, el uso del teatro y otras manifestaciones artísticas como vehículo trasmisor del mensaje medioambiental.

En las Jornadas de Siembra, el voluntario no solo aprende los beneficios que aporta al medioambiente la recuperación de la capa boscosa, forma correcta de siembra entre otros valiosos conocimientos, sino que dirige a otros participantes y miembros del voluntariado a tomar conciencia de las situación y realizar una siembra efectiva. Por otra parte, en las Jornadas de Limpieza de playa, el asistente a las mismas, se percata de la gran cantidad y tipos de desperdicios que son arrastrados por las corrientes marinas y depositados en las playas con el daño que ocasiona a los ecosistemas, y se promueve que este voluntario aporte soluciones que reviertan esos daños. Esta labor del voluntario, cuyo desempeño no le genera beneficios económicos, desarrolla la sensibilidad respecto a la problemática medioambiental que se trabaje, permitiéndole percibir las soluciones que su propia conducta puede generar o la manera en que su labor ayuda a que otros las generen y sirviendo de orientador o líder a los grupos de trabajo. Realmente, la actividad es transformadora.

Dentro de la estructura organizativa del voluntariado se encuentran el coordinador del programa, los estudiantes líderes de grupo y otros miembros. Los líderes de grupo son aquellos que, por la experiencia que tienen al participar en diversas actividades del voluntariado, desarrollan habilidades y destrezas que les permiten dirigir y ayudar a los que recién se inician o precisan de orientación. De esta manera, la interacción entre los miembros del grupo desarrolla y fortalece las competencias sociales, comunicativas, los valores institucionales, el pensamiento analítico y crítico, el trabajo colaborativo y la identificación con la institución.

Para la formación del voluntariado en sus primeras etapas, fue fundamental el conocer la vida estudiantil en el campus, la cultura institucional, tomar en cuenta los periodos académicos para la programación de las actividades y la estrategia adecuada de comunicación con las áreas académicas y operativas, facilitadoras de la implementación de actividades, para minimizar la resistencia al cambio en los procesos y responsabilidades que el proyecto involucraba. Las lecciones aprendidas al inicio, permitieron una rápida implementación de mejoras, tanto en diseño y programación de las actividades, como en los procesos internos institucionales del programa Pienso en Verde.

Dentro de los voluntarios hay un grupo que forman los edecanes de Pienso en Verde. Ellos dan apoyo a otras áreas o instituciones en la realización de actividades medioambientales. Así mismo, dentro del programa está el grupo de teatro Mimos de Pienso en Verde, cuyas presentaciones usualmente se realizan en fechas del calendario medioambiental y se basan en llevar un montaje con un mensaje medioambiental.

En una investigación descriptiva basada en encuesta realizada a un grupo de 48 voluntarios medioambientales de la UNPHU, el 75\% consideró que pertenecer a una universidad que desarrolla voluntariado y actividades en beneficio del medioambiente es muy importante, y que es un aspecto diferenciador respecto a otras universidades. Cerca del 92\% opinó que el ser voluntario de Pienso en Verde ayudaba a su formación profesional; el 100\% piensa que la UNPHU, a través de su programa Pienso en verde, orienta a los estudiantes a cuidar el medioambiente. $\mathrm{Y}$ en cuanto a elegir una o varias de las cosas que más le gustaban de ser voluntario, el $83 \%$ eligió el proteger al medioambiente, casi el $67 \%$ seleccionó la op- 
ción de saber que ayuda a una buena causa y alrededor del $64 \%$ optaron por aprender a hacer algo diferente.

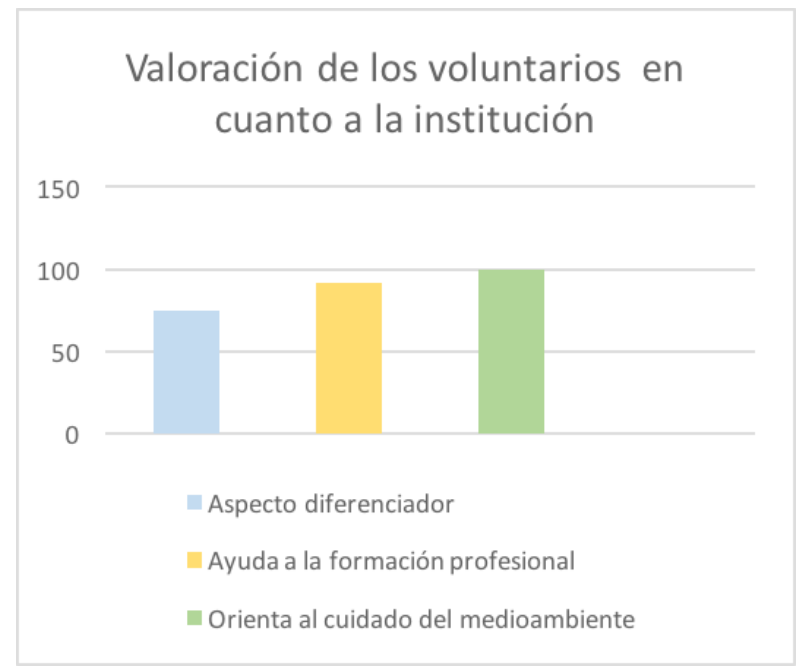

Cabe destacar que el 50\% encuentra, entre lo que más le gusta de ser voluntario, ser parte activa de la universidad. Este aspecto es fundamental a ser tomado en cuenta, tanto para fortalecer el vínculo con la universidad como para la permanencia en el voluntariado, teniendo un tercio de los encuestados de uno a dos años en el voluntariado y un $50 \%$ más de dos años.

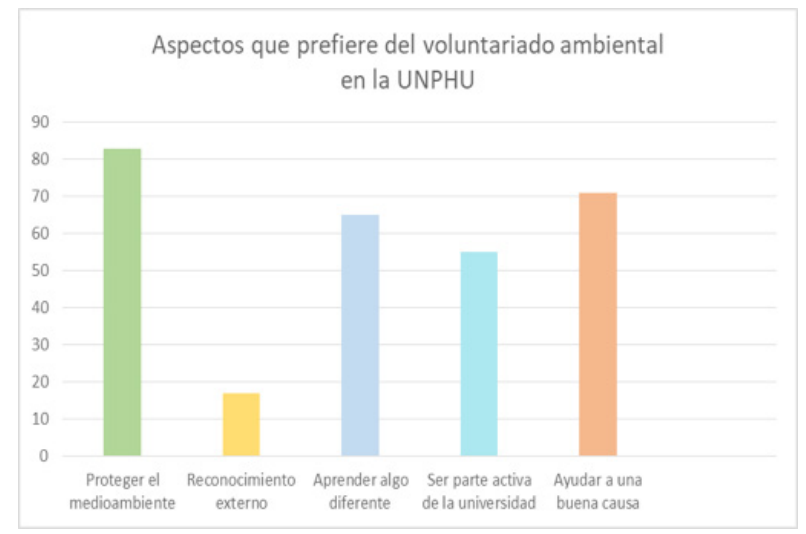

En la parte formativa, se cuenta con la asignatura optativa Conciencia Ambiental, en la modalidad presencial y semipresencial, que abarca los conceptos fundamentales de ecología

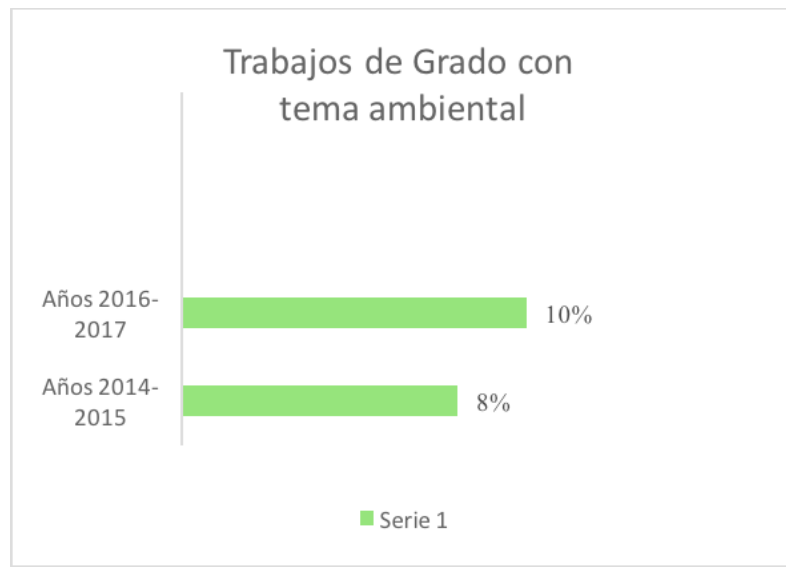

y medioambiente, así como principales temas ambientales de la actualidad, relacionando los conceptos y temas a la vida universitaria y la comunidad. En postgrado, se ofertan tres programas relacionados con el medioambiente. Por otro lado, casi todos los programas educativos de grado poseen una asignatura específica que vincula el medioambiente a la carrera, además de la realización de diplomados y talleres orientados al cambio climático y otras problemáticas, cursos, conferencias, seminarios internacionales, foros y congresos, con el tema medioambiental como parte principal. De igual forma, a lo largo del año, se realizan a través de las redes sociales, campañas de sensibilización o concientización acerca de temas ambientales.

En cuanto a los proyectos de investigación, del 2014 al 2015, alrededor del 8\% de los trabajos de tesis presentados tenían un enfoque medioambiental, incrementado este porcentaje a un $10 \%$ aproximadamente entre los años 2016 y 2017. Esto nos puede indicar que efectivamente se está logrando una mayor sensibilidad y conciencia en el egresado, en cuanto al tema medioambiental, pero es necesario realizar un estudio más profundo que muestre valores precisos y tendencias firmes en ese sentido. Es un reto poder cuantificar los aportes que este tipo de formación ofrece, además, en la parte de innovación y emprendimiento, reflejado en proyectos de investigación que aportan soluciones a problemáticas ambientales. 
Al momento de implementar los proyectos ambientales, es muy importante contar con personal de apoyo entrenado, tanto en una cultura de servicio, como en el dominio de los beneficios que su labor implica para el ambiente y para la institución. En base a la experiencia de este quehacer, aunque es común que se encuentre cierta resistencia al cambio de procesos y procedimientos; en la UNPHU la constancia y una supervisión constante y cercana, así como el involucramiento de estos departamentos al momento de diseñar las políticas de mejoras y nuevos proyectos, favorece el éxito del logro de los objetivos. Sólo un fuerte respaldo de las autoridades universitarias puede lograr este cambio de cultura y junto a la persistencia, la definición de objetivos claros y la visión de la mejora continua en todas las áreas, permitirá un desarrollo en consonancia con los objetivos estratégicos institucionales.

\section{Para reflexionar y líneas a seguir}

La UNPHU ha mostrado un crecimiento de alrededor de un $39 \%$ de aumento de su matrícula, entre los años 2005 y 20016, según muestra el Ministerio de Educación Superior, Ciencia y Tecnología, en su reporte de estadísticas publicado este año, lo que indica que la dirección que ha elegido para desarrollarse es percibida por los estudiantes que la prefieren.

Los resultados de la encuesta coinciden con lo señalado por Penner (2002) en cuanto al grado de integración del voluntario con la institución, así como con Chacón y Dávila (2001) en cuanto a la valoración del aspecto medioambiental alineados con sus valores personales. Vale la pena estudiar con mayor profundidad el nivel de satisfacción del voluntario medioambiental, en nuestro país, más allá de las tres variables que mencionan Vecina et al, (2009).
La responsabilidad ambiental en las universidades ha sido y es objeto de estudio, pues todavía se desconoce de manera cuantificable el efecto beneficioso que esta produce en las comunidades, internas y externas de la academia, siendo estos beneficios muchas veces intangibles y verificables a corto mediano y largo plazo, lo que impide valorar de manera efectiva su alcance e impacto. La falta de información para el público, de un plan concreto o de políticas medioambientales claras, congruentes con la filosofía de estas instituciones, puesta a disposición de los interesados a través de medios de comunicación accesibles, no permite percibir el trabajo que se hace y se puede hacer desde las universidades en República Dominicana. Y este es un tema que puede desarrollarse a profundidad.

Hay que tomar en cuenta que el esfuerzo en desarrollar una RAU, característica de cada institución, conlleva el uso de recursos cuyo monto dependerá del tipo y el alcance de cada proyecto y programa que se desarrolle, considerando que el aporte del estado hacia las universidades es muy bajo. Es favorable la realización de alianzas con instituciones de diferentes países para generar propuestas ante problemáticas similares.

En la UNPHU, independientemente de otras áreas en las que desarrolla su RSU, la que corresponde a la ambiental posee un programa claro y definido. Se puede percibir en la mejora sustancial de su imagen institucional, la integración del estudiantado a las actividades, tanto dentro del voluntariado como fuera de él, el nivel de satisfacción en la labor que realizan, el reconocimiento al valor agregado que el voluntariado le ofrece como profesional y persona, de igual forma en los trabajos de grado y las áreas de gestión. Todo esto relacionado a su vez con varios aspectos de la END 2030, especialmente en sus Ejes Estratégicos 3 y 4, del 
mismo modo en el logro de algunos de los ODS como por ejemplo el siete, 11,13,17, relacionados con Energía Limpia y No Contaminante, Ciudades y Comunidades Sostenibles, Acción por el Clima y Alianzas para lograr Objetivos. El resultado es definitivamente beneficioso, y merece sistematizarse y replicarse.

Se hace necesario realizar estudios que midan con mayor profundidad el impacto de ese tipo de responsabilidad universitaria en la formación de los que egresan, que de forma medible contextualicen el perfil de nuestro voluntariado medioambiental; que la experiencia en la UNPHU pueda servir de base para que otras instituciones repliquen las buenas prácticas, uniendo esfuerzos como país en una misma dirección. Es importante verificar cómo los egresados, que serán los tomadores de decisiones a nivel de empresas o país, realmente se impregnan de esta forma de ayudar que beneficia a todos y provoca el cambio positivo que el mundo precisa.

\section{Referencias}

Cone Comunications \& Ebiquity (2015).Global CSR Study. [Estudio global de CRS]. Recuperado de URL: http://www.conecomm. com/2015-cone-communications-ebiquity-global-csr-study-pdf/

Cone Comunications \& ECHO (2013).Global CSR Study. [Estudio global de CRS]. Recuperado de URL: http://www.conecomm.com/2013-cone-communicationsecho-global-csr-study-pdf

Chacón, F., y Dávila, M. (2001). Diferencia entre el perfil de voluntariados ecologistas y socioasistenciales. Revista Intervención Psicosocial X (2), 137-153. Recuperado de URL: http://eprints.ucm. es/40517/1/76933.pdf
De Sousa Santos, B. (2007). La Universidad en el siglo XX1 para una reforma democrática y emancipatoria de la universidad. Recuperado de URL:http://www. boaventuradesousasantos.pt/media/ universidad_siglo_xxi-.pdf

Eschenhagen, M. (2011).El tema ambiental y la educación ambiental en las universidades: algunos indicadores y reflexiones. Revista de educación y desarrollo IXX (19),35-41. Recuperado de URL: http:// pensamientoambiental.de/wp-content/ uploads/2014/10/el-tema-ambiental-y-la-educacion.pdf

Fernández, G. R. (2013). La dimensión económica del desarrollo sostenible. San Vicente, Alicante. Editorial Club Universitario. Recuperado de : https://ebookcentral.proquest.com

García-Ayuso, M., y Larrinaga, C. (2004). El lado oculto de los intangibles: activos y pasivos ligados a la sostenibilidad. Recuperado de URL: https://ebookcentral. proquest.com

Instituto Internacional para la Educación Superior en América Latina y el Caribe IESALC UNESCO. Recuperado de URL: http://www.iesalc.unesco.org. ve/index.php?option $=$ com_content\&view $=$ article\&id=79:responsabilidad

Ley 61-13. Recuperado de URL:

http://economia.gob.do/mepyd/ wp-content/uploads/archivos/asfl/ marco-legal/ley-61-13-regimen-juridico-voluntariado-en-republica-dominicana.pdf 
Ministerio de Educación Superior, Ciencia y Tecnología (20017). Informe General sobre Estadísticas de Educación Superior 2016 y Resumen Histórico 20052016. Recuperado de URL: mescyt. gob.do/transparencia/.../estadisticas-institucionales?...informe-de-estadistica-2016

Organización de las Naciones Unidas. Consejo Económico Social. (2017). Progresos en el logro de los Objetivos de Desarrollo Sostenible Informe del Secretario General. Recuperado de URL:http:// www.un.org/en/ga/search/view_doc.asp?symbol=E/2017/66\&referer=http:// www.un.org/es/documents/index.htm1\&Lang $=\mathrm{S}$

Organización de las Naciones Unidas para la Educación, la Ciencia y la Cultura UNESCO (2016). Informe de seguimiento de la educación en el mundo 2016: La educación al servicio de los pueblos y el planeta. Creación de futuros sostenibles para todos. Recuperado de URL : http://unesdoc.unesco.org/images/0024/002485/248526S.pdf

Penner, L.A. (2002). Dispositional and organizational influences on sustained volunteerism: [Influencias disposicionales y organizativas sobre el voluntariado sostenido: Una perspectiva intereccionista]. An interactionist perspective. Journal of Social Issues, 58(3), 447-467.Recuperado de URL: http://nclc203wagner. pbworks.com/f/Demographic.pdf
Preziosa, M. (2005). La definición de «Responsabilidad Social Empresarial» como tarea filosófica. Journal of Economics, Finance and Administrative Science, 10 (18-19), 39-59. Recuperado de URL:http://www.redalyc.org/ pdf/3607/360733600002.pdf

Programa de Voluntarios de las Naciones Unidas (VNU), 2011. Recuperado de URL: http://www.undp.org/content/undp/es/ home/librarypage/capacity-building/ Volunteerism_Report_2011.html

Senior, A., Narváez, M., Fernández, G., y Revilla, J. (2007). Responsabilidad ambiental: factor creador de valor agregado en las organizaciones. Revista de Ciencias Sociales (Ve), XIII (3), 484494. Recuperado de URL: http://www. redalyc.org/pdf/280/28011681009.pdf

Takayanagui, A. D., y de la Fuente, J. R. (2015). Perspectives about the university's social engagement. [Perspectivas sobre el compromiso social de la universidad]. La responsabilidad social, 33. http://unesdoc.unesco.org/images/ 0024/002442/244270m.pdf

Vallaeys, F. (2008). Responsabilidad Social Universitaria: una nueva filosofía para la gestión ética e inteligente de las universidades. Revista ESS Educación Superior y Sociedad. Año XIII (2), 209 Recuperado de URL: http:// pensamientoambiental.de/wp-content/ uploads/2014/10/el-tema-ambiental-y-la-educacion.pdf 
Vecina Jiménez, M., Chacón Fuertes, F., y Sueiro Abad, M. (2009). Satisfacción en el voluntariado: estructura interna y relación con la permanencia en las organizaciones. Revista Psicothema XXI, (1), 112-117. Recuperado de URL: http://www.psicothema.com/pdf/3603.pdf
Worldwatch Institute. (2015). Aprovechamiento de los Recursos de Energía Sostenible de la República Dominicana. Recuperado de URL: http://www.worldwatch. org/system/files/DR_report_Spanish hi-res.pdf

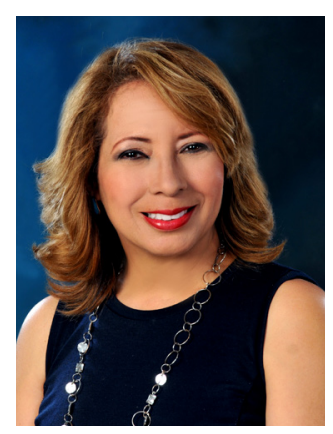

Dolly Martínez

Graduada de Ingeniería Civil y de Agrimensura en la Universidad Nacional Pedro Henríquez Ureña. Posee grado de Magister en Administración de Negocios de la Pontifica Universidad Católica Madre y Maestra PUCMM, además de Magister en Educación Superior y Gestión Universitaria de la Universidad Católica de Santo Domingo. Con un ejercicio de más de veinte años en el área de la ingeniería civil, fundamentalmente en el sector privado, se apasiona por la docencia universitaria y el cuidado del medioambiente, como ejes transformadores y guías del desarrollo humano. Ha recibido durante más de cinco años, formación continua en el área ambiental. En el campo de la educación, ha impartido docencia en la UNPHU y PUCMM. Actualmente es Directora Ambiental, docente y miembro de la Unidad de Calidad Académica de la Escuela de Ingeniería Civil de la UNPHU. 